\title{
Real time pitch detection using a digital signal processor
}

\author{
Shogo Nakamura, Michiharu Mito, Kiyoshi Takahashi, \\ Yoshihiko Horio, and Tadahiro Kubota \\ Department of Electronic Engineering, Tokyo Denki University, \\ 2-2, Nishiki-cho, Kanda, Chiyoda-ku, Tokyo, 101 Japan
}

(Received 20 February 1989)

\begin{abstract}
Pitch detection is an essential technique in the various speech processing systems. The pitch period is useful for applications such as the speaker recognition, the speech synthesis and so on. A new frequency-time domain method in order to obtain a fundamental frequency of speech is presented in this paper. The paper also describes the results of the computer simulations and a real time pitch period detector using a conventional Digital Signal Processor. The algorithm uses the Discrete Running Fourier Series (DRFS) for emphasis of the peaks of the speech signal corresponding to the pitch and a new simple rule for the determination of the pitch period.
\end{abstract}

PACS number: 43. 72. Ar

\section{INTRODUCTION}

Pitch detection is an essential technique in the various speech signal processing systems. The pitch period is useful for speaker recognition, speech synthesis and so on. The pitch detection algorithms usually use the correlations or spectra of speech time signals. But it is difficult to obtain the pitch frequency in real time using their algorithms. ${ }^{1,2)}$ A method which obtains the time intervals between successive peaks associated with the pitch from the speech time signal seems to be suitable for real time processing. However, since the speech signals usually include many peaks not associated with the pitch, a technique to identify the peaks corresponding to the pitch is needed. Accordingly, it is important to determine correctly peaks which are caused by the pitch. Although the proposed algorithm is basically performed in the time domain, the Discrete Running Fourier Series (DRFS) which gives a kind of short time Fourier spectrum is used in order to facilitate the determination of peaks associated with the pitch. Then the pitch period is obtained by measuring intervals between the successive peaks corresponding to the pitch. Thus, this algorithm consists of two parts. One is the processing of the peak emphasis as was stated previously. The other is the pitch period detection. This estimates the intervals between successive peaks that are associated with the pitch by a novel decision rule.

\section{PEAK EMPHASIS}

A speech waveform caused by glottal vibrations includes many peaks that are not associated with the pitch. This makes the determination of the accurate peaks difficult. So it is necessary to emphasize the peaks corresponding to the pitch. The emphasis is realized by a DRFS which gives a short time spectrum and a proper window processing. A speech signal is sampled at $10 \mathrm{kHz}$ and filtered by a low pass filter whose cutoff frequency is $2 \mathrm{kHz}$. Then the sampled data are weighted by a 10-point edge emphasis-window which has $1 \mathrm{~ms}$ interval. This window is expressed in Eq. (1) and its shape in the time domain is plotted in Fig. 1. Although Eq. (1) resembles the well-known Hanning window, this has a quite different property. In 


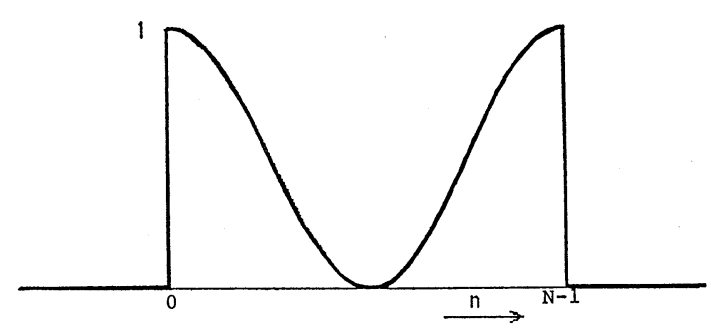

Fig. 1 An edge emphasized window.

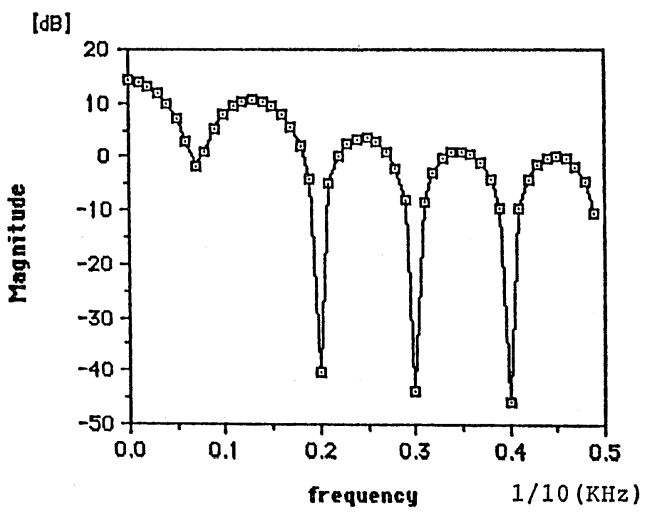

(a)

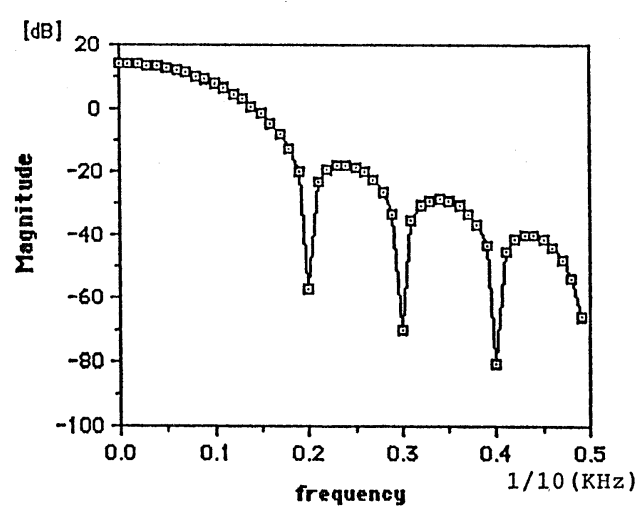

(b)

Fig. 2 Frequency characteristics. (a) edge emphasized window (b) Hanning window.

order to compare this window with the usual Hanning window, frequency characteristics of both windows are shown in Fig. 2.

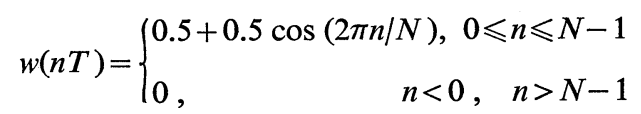

If there is a large peak in the vicinity of the edges of the window, this will cause leakages into a few channels of DRFS. In this case, the peak amplitude of the output time signal which is synthesized by the DRFS will be emphasized.

Here, we briefly describe DRFS. ${ }^{3)} N$ points DRFS is defined by

$$
F(n, m)=\sum_{k=0}^{N-1} f(n+k) W^{-m \pi k}, \quad W=e^{i 2 \pi / N}
$$

where $n$ is a time index and $m$ is a frequency index.

The next relation holds between $F(n, m)$ and $F(n-1, m)$.

$$
\begin{aligned}
F(n, m)= & {[F(n-1, m)+f(n+N-1)} \\
& -f(n-1)] W^{m}
\end{aligned}
$$

This equation can be realized by a comb and a simple recursive filters as shown in Fig. 3.

A reconstructed time signal can be synthesized by all frequency components of the DRFS. Here, only $F(n, 0)$ and $F(n, 1)$ are used because of the signal being band-limited within $2 \mathrm{kHz}$. The synthesized output signal can be obtained by the following relation;

$$
O(n)=\sum_{m=0}^{1} a_{m} \sqrt{a_{m}^{2}+b_{m}^{2}}
$$

where $a_{m}$ and $b_{m}$ represent a real and an imaginary parts of each output of the DRFS respectively. $O(n)$ preserves a sign of an original speech signal since the Eq. (4) represents a summation of a real component of power, $a_{m}{ }^{2}+b_{m}{ }^{2}$. As a result, the emphasized peaks occur on the two sides of positive and negative levels following the original speech

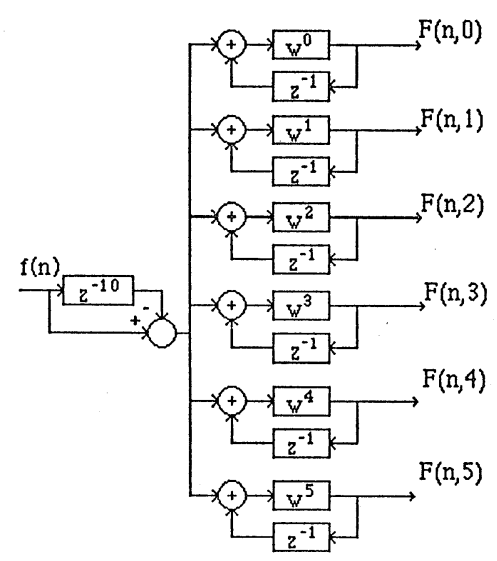

Fig. 3 A block diagram DRFS. 


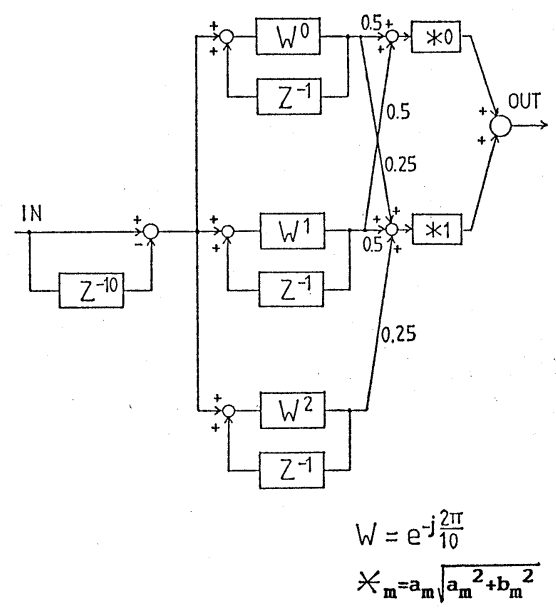

Fig. 4 A block diagram emphasizing peaks.

signal and this property is used in the pitch estimation.

A block diagram of the above algorithm is shown in Fig. 4 and a computer simulation is also represented in Fig. 5. In order to compare this with the other method, a waveform which has been emphasized by a squared way is also shown in Fig. 6 . It is clearly noticed that the proposed way is better than the squared one.

\section{PITCH ESTIMATION}

The pitch period is detected by obtaining an interval between the successive peaks which are emphasized. Since a speech signal includes rather complicated several peaks, the peaks which do not correspond to the pitch have to be rejected. We have proposed a simple rule for this purpose. This rule is summarized below.

1) It is assumed that the pitch peaks appear on the two sides of positive and negative levels in a speech time signal.

2) A peak on a side where the difference between the largest peak and the next one is larger than that of the other side is adopted as a correct peak.

3) If a new candidate of the pitch frequency is within $70 \sim 130 \%$ of the previous one, the pitch frequency is renewed by the new candidate.

4) An initial value of pitch frequency is obtained as an average value of the beginning three periods.

In addition to these rule, a peak hold with some leakage is used to search the accurate peaks. The
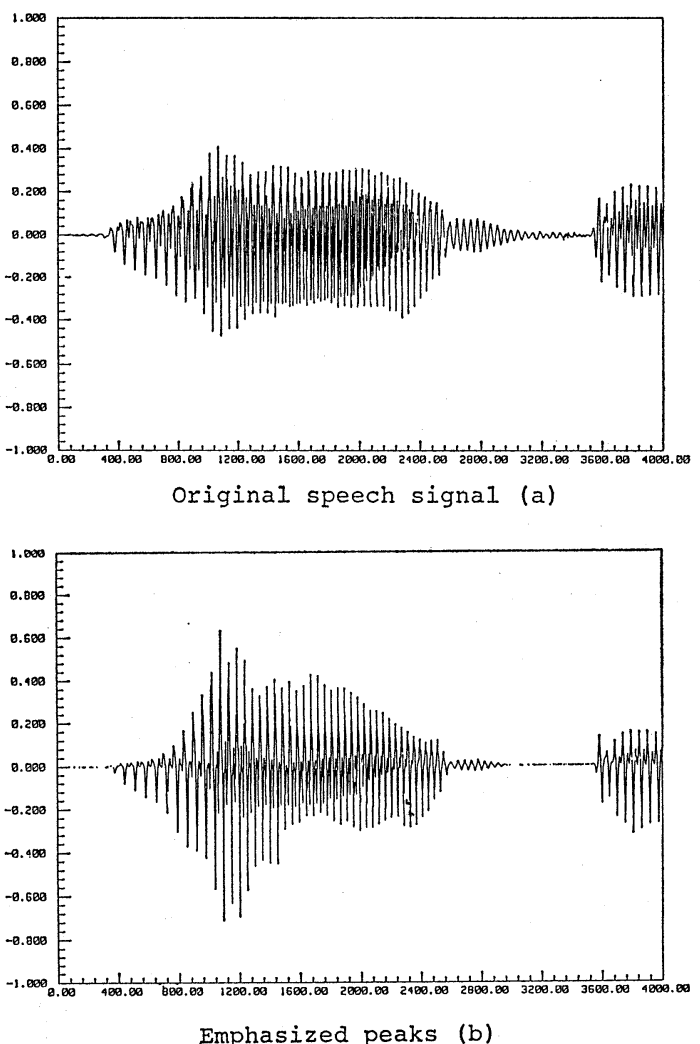

Fig. 5 An example of the peak emphasized signals.

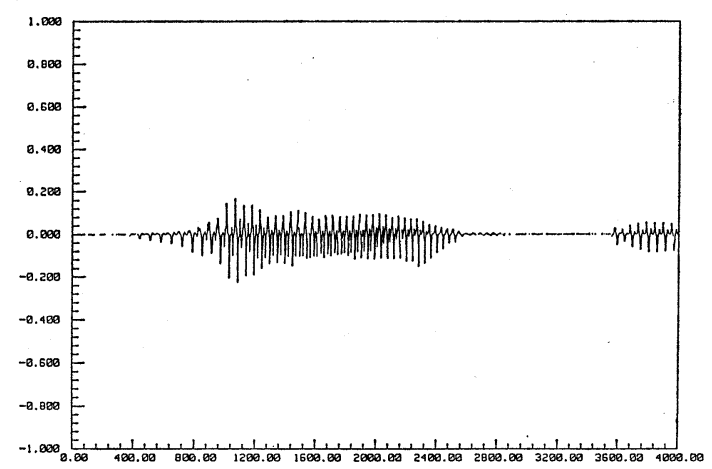

Fig. 6 An example of the emphasized signal by squaring method.

peak value $R_{\max }$ is attenuated exponentially such as;

$$
r(n)=R_{\max } \exp (-0.02 n)
$$

where $n$ is the number of sampled points from the top of a peak. The peak smaller than $r(n)$ will not 


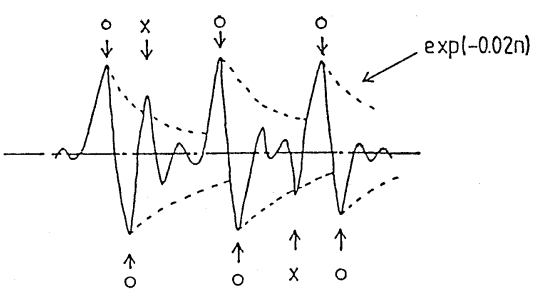

Fig. 7 An explanation of peak detection rule.

be considered as a peak. An example of a peak determination is shown in Fig. 7. In Fig. 7, $\bigcirc$ represents accepted peaks and $\times$ shows rejected peaks by the condition of rule (3). Finally, the pitch period is obtained as a successive interval of the accepted peaks as mentioned before.

\section{A REAL TIME PITCH DETECTOR}

A conventional digital signal processor $\mu \mathrm{PD} 7720$ by NEC has been used to realize a pitch detector. ${ }^{4,5)}$ The algorithm consists of two parts and each part uses one DSP. Peaks of a sampled speech signal are emphasized by the first DSP and the resultant data are sent to the second DSP in order to obtain the pitch period. The second DSP counts the number of sampled points between the peaks and delivers it to an 8-bit D-A converter. The output of this pitch detector is given by a voltage associated with the pitch period. A hardware block diagram is shown in Fig. 8. The algorithm has been applied to display intonations of speech utterances. ${ }^{6}$ ) Figure 9 shows intonational waveforms of utterances "He is an able man." In the figure, upper dashed curve represents an intonation of utterance by a native male speaker and lower one represents an intonation by a Japanese male speaker. We also examined different 50 English sentences which had been pronounced by native speakers. As a result of investigating them by eye-measurements, we

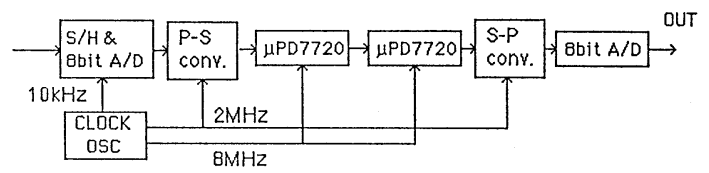

Fig. 8 A block diagram of the proposed pitch detector.

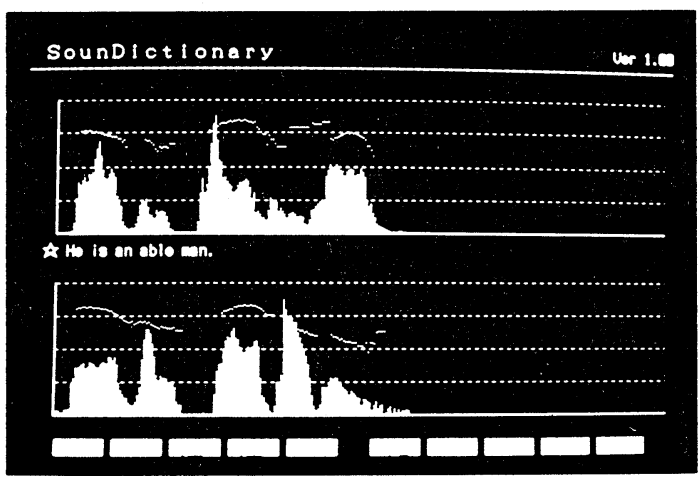

Fig. 9 An application to display intonations of speech signals.

could satisfactorily get a pitch extraction error within $4.53 \%$.

\section{CONCLUSION}

This paper describes a simple real time pitch detection algorithm and the real time pitch detector by using a conventional DSP. In the proposed method, a pitch period is detected by obtaining intervals between the peaks which correspond to the pitch. Many traditional methods detect the pitch period as the average of a specific time span, but this method can detect it instantaneously.

The realized pitch detector behaves correctly except the case where speech signals are unstable at the beginning. So, an accurate way to determine the initial pitch value is required. Furthermore, it is also necessary from the point of view of a practical system that this algorithm would be realized into a single DSP.

\section{ACKNOWLEDGMENT}

This work was supported by Center for Research, Tokyo Denki University.

\section{REFERENCES}

1) L. R. Rabiner, M. J. Cheng, A. E. Rosenberg, and C. A. Mcgonegal, "A comparative performance study of several pitch detection algorithms," IEEE Trans. Acoust. Speech Signal Process. ASSP-24, 399-418 (1976).

2) L. R. Rabiner and R. W. Schafer, Digital Processing of Speech Signals (Prentice-Hall, Englewood Cliffs, (1978).

3) A. Papoulis, Signal Analysis (McGraw-Hill, New York, 1977). 
S. NAKAMURA et al.: REAL TIME PITCH DETECTION USING A DSP

4) K. Takahashi, S. Nakamura, and T. Kubota, "An approach of displaying intonation," IEICE Natl. Conf. Rec. Part 7, No. 241 (1988) (in Japanese).

5) M. Mito, K. Takahashi, S. Nakamura, and T. Kubota, "Real time pitch detection using DSP,"
IEICE Natl. Conf. Rec. Part 1, 162 (1987) (in Japanese).

6) S. Nakamura, T. Kubota, and M. Ohuchi, "A realization of an English dictionary with voice," IEICE J72-A, 977-984 (1989) (in Japanese). 